Apidologie, 1973, 4 (1), 45-55.

\title{
DEUX MÉTHODES COLORIMÉTRIQUES SIMPLES PERMETTANT D'APPRÉCIER LA TENEUR EN GLUCOSE ET EN FRUCTOSE DES MIELS
}

\section{Zwei einfache kolorimetrische Methoden zur Bestimmung des Glukose- und Fruktosegehaltes von Honigen.}

\author{
Michel GONNET \\ avec la collaboration technique de Fernande Paris \\ Station expérimentale d'Apiculture, \\ Centre de Recherches agronomiques d'Avignon, I. N. R. A., \\ 84140 Montfavet
}

\author{
SUMMARY \\ TWO SIMPLE COLORIMETRIC METHODS TO ESTIMATE THE GLUCOSE AND \\ FRUCTOSE CONTENT IN HONEYS
}

A titration method of reducing sugars in honey was perfected. This method can be used in the practical work conditions of a beekeeping cooperative.

Glucose is titred with a colorimetric method which uses the benzidine in acetic medium; the fructose titration is accomplished with a similar method, which requires the use of $B$ indolyl-acetic acid in chlorhydric medium. Reference titrations were accomplished with gaschromatography.

The results obtained are satisfying with regard to the colorimetric analysis of glucose, a little less accurate for the fructose. Nevertheless these methods supply a good estimation of the reducing sugars content (glucose and fructose) in an honey. This estimation is usually enough for the routine analysis in the practical field, but cannot be taken into account as an official reference method.

\section{RESUMÉ}

L'auteur a mis au point une méthode de dosage des sucres réducteurs dans le miel; cette méthode est utilisable dans les conditions pratiques de travail d'une coopérative apicole par exemple.

Le glucose est dosé par une méthode colorimétrique utilisant la benzidine en milieu acétique; le dosage du fructose est effectué par une méthode analogue nécessitant l'emploi d'acide $\beta$ indolyl - acétique en milieu chlorhydrique. Les dosages de référence ont été effeetués par chromatographie_en phase gazeuse. 
Les résultats obtenus sont satisfaisants en ce qui concerne l'analyse colorimétrique du glucose, un peu moins précis pour le fructose. Ces méthodes autorisent néanmoins une bonne estimation de la teneur en sucres réducteurs (glucose et fructose) dans un miel. Cette estimation suffit le plus souvent dans le domaine pratique pour des analyses de routine, mais ne peut être prise en considération comme méthode de référence officielle.

\section{I. - INTRODUCTION}

Le glucose et le fructose sont deux monosaccharides réducteurs qui entrent en moyenne pour $70 \%$ dans la composition d'un miel. La proportion de l'un par rapport à l'autre est assez variable, mais le fructose est le plus souvent dominant. Les moyennes calculées sur 490 miels américains de différentes provenances sont de $38 \%$ de fructose et $31 \%$ de glucose. (White J. et al. 1962.)

En pratique, le rapport glucose/fructose est assez difficile à déterminer avec exactitude par l'analyse courante; différentes méthodes ont cependant été proposées dans ce but. White J. et Maher J., (1954) font une séparation sur colonne en utilisant une phase fixe, la poudre de charbon, et une phase mobile, un mélange éthanol/eau. Ils dosent ensuite les sucres dans les fractions recueillies par les méthodes chimiques appropriées. Cette technique est bonne, sans doute, mais l'analyse est longue et de ce fait ne peut être retenue pour les examens de routine.

Pourtallier J. (1967) a adapté aux miels une méthode de dosage des sucres par chromatographie en phase gazeuse. C'est une technique très sûre et très complète mais là aussi la préparation de l'analyse exige beaucoup de temps; de plus l'appareillage requis est onéreux.

Les analyses chimiques courantes permettent une évaluation du pourcentage des différents sucres contenus dans une solution de miel. Le dosage iodométrique des aldoses, par exemple, (méthode de BougauLT) est une analyse simple, d'exécution rapide et qui nécessite peu de matériel. On peut traduire facilement les résultats obtenus en glucose, mais de manière assez approximative car certains dissaccharides du miel réagissent en partie, de même d'ailleurs qu'une proportion non négligeable du fructose comme nous le confirmons dans ce travail. Néanmoins, en l'absence d'autres méthodes, on peut dire que les résultats obtenus constituent une approximation intéressante pour le technicien.

Les dosages réductimétriques (type Felhing ou Bertrand) sont également applicables mais les résultats fournis sont globaux; ils révèlent l'ensemble des sucres réducteurs du miel. La polarimétrie, complément normal de ce type d'analyse pour définir les taux respectifs de glucose et de fructose contenus dans une solution de sucre interverti, est ici inutilisable car certains sucres mineurs du miel dévient fortement la lumière polarisée et faussent l'interprétation des résultats.

Des méthodes de chromatographie sur papier (Maurizio A., 1959) et par 
chromatographie sur gel de silice (Pourtallier J., 1964) ont été décrites. Elles paraissent convenir assez mal à des examens de routine.

La préparation du miel pour la commercialisation dans les conditions actuelles exige des traitements particuliers qui doivent garantir la parfaite conservation du produit. La mise en œuvre de procédés technologiques importants peut être requise à cet effet. Il est alors nécessaire d'opérer un choix dans les miels dont on dispose si l'on veut obtenir des lots de qualité constante. Ce choix repose le plus souvent sur la connaissance de trois données essentielles : la teneur en glucose, en fructose et en eau.

La cristallisation des miels est d'une manière générale très liée aux rapports glucose/fructose et glucose/eau. Pour les traitements à appliquer dans le domaine de la cristallisation dirigée, par exemple, il faut tenir compte de ces paramètres essentiels.

Mais il y a d'autres opérations technologiques qui ne peuvent pas être conduites dans des conditions parfaites si l'on ne connaît pas le taux de glucose ou celui de fructose dans le miel que l'on veut traiter : c'est le cas pour la pasteurisation. Quand on pasteurise un miel, c'est principalement pour détruire les cristaux de glucose et assurer ainsi le maintien du produit à l'état liquide; il est important dans ce cas de connaître la teneur en glucose avant d'entreprendre le traitement. Une pasteurisation pratiquée sur un miel ayant un pourcentage de glucose élevé risque de s'avérer inutile et même néfaste, car la recristallisation du produit sera généralement rapide et la granulation très grossière. Une telle opération est donc à éviter.

Pour ces raisons, il est souhaitable que quelques analyses simples puissent être pratiquées sur les lieux mêmes de conditionnement du miel. C'est pourquoi nous avons mis au point deux méthodes d'analyse, l'une du glucose, l'autre du fructose, suffisamment précises et rapides pour qu'elles puissent être utilisées dans la pratique par le laboratoire d'une coopérative apicole ou d'un négociant en miel. L'appareillage requis pour ces analyses n'est pas très important; il est d'ailleurs identique à celui qui est employé pour d'autres analyses classiques de contrôle de la qualité des miels, tels que le dosage de l'HMF ou les déterminations de l'activité diastasique. De telles analyses sont couramment pratiquées dans ce type de laboratoires de contrôles.

Les méthodes qui ont retenu notre attention et que nous avons adaptées à l'analyse des miels sont des micro-méthodes colorimétriques. Nous nous sommes inspirés pour le dosage du glucose d'une méthode décrite en 1954 par Jones et Pridham et, pour le dosage du fructose, d'une autre méthode mise au point en 1956 par Herovsky. Ajoutons, que l'analyse colorimétrique appliquée aux dosages des sucres dans le miel n'est fiable que lorsqu'elle est conduite dans des conditions expérimentales précises. Nous nous sommes donc attachés tout d'abord à définir ces conditions et à tester le degré de spécificité des méthodes que nous avons mises au point. 


\section{II. - DESCRIPTION DES MÉTHODES}

\section{A. 一 Préparation d'une solution de sucres témoins}

\section{a. Principe}

Il s'agit d'une solution de référence de titre connu; elle sert d'étalon pour les deux types de dosages. Elle contient les sucres réducteurs, glucose et fructose, en quantités égales; elle est sensiblement comparable à une solution de miel équivalente.

\section{b. Réactifs}

- Acide chlorhydrique concentré pur.

- Saccharose chimiquement pur.

\section{c. Mode opératoire}

On pèse $1,900 \mathrm{~g}$ de saccharose que l'on met en solution dans $45 \mathrm{ml}$ d'eau distillée. Sur cette solution on pratique une intervention du type CLERGET, c'est-à-dire que l'on ajoute à la solu. tion de sucre $5 \mathrm{ml}$ d'HCL. On chauffe lentement le mélange au bain marie de manière à atteindre $72^{\circ} \mathrm{C}$ en 10 minutes. On laisse refroidir spontanément et on amène à $100 \mathrm{ml}$ en fiole jaugée. Cette solution mère contient exactement $20 \mathrm{mg}$ de sucres réducteurs par $\mathrm{ml}$. On en prélève avec précision $2 \mathrm{ml}$ que l'on amène à $100 \mathrm{ml}$ avec de l'eau distillée. On utilise cette seconde dilution comme témoin pour les deux types d'analyses.

\section{B. - Recherche du glucose}

\section{a. Principe}

Dans les conditions expérimentales décrites ci-dessous, on fait réagir le glucose contenu dans une solution de miel avec de la benzidine en milieu acétique concentré. On obtient une coloration jaune-orangée d'une intensité proportionnelle à la concentration du glucose dans le milieu.

\section{b. Réactifs}

On prépare une solution de benzidine à $0,2 \%$ dans de l'acide acétique pur; le mélange est homogénéisé par agitation magnétique pendant 2 minutes environ. On ajoute alors $0,1 \%$ de chlorure d'étain broyé au mortier et l'on poursuit encore l'agitation pendant 5 minutes après l'apparition d'un trouble dans la solution. On filtre le mélange. Le réactif doit être limpide; il est renouvelé journellement.

\section{c. Mode opêratoire}

On prépare une solution à $2 \%$ de miel dans de l'eau distillée. De cette solution on prélève avec précision $2 \mathrm{ml}$ que l'on amène à $100 \mathrm{ml}$ en fiole jaugée. Cette dernière dilution est à $4.00 \mu \mathrm{g}$ de miel par ml.

— Dans un tube à essai on prélève $0,75 \mathrm{ml}$ de la solution de sucre de référence (soit $150 \mu \mathrm{g}$ de glucose); on complète à $1 \mathrm{ml}$ avec de l'eau distillée. Dans un autre tube on écoule $1 \mathrm{ml}$ de la solution diluée de miel (soit $400 \mu \mathrm{g}$ ). Un témoin à blanc est réalisé avec $1 \mathrm{ml}$ d'eau distillée. On utilise pour tous ces prélèvements de précision, des pipettes étalons de $1 \mathrm{ml}$ graduées par $0,05 \mathrm{ml}$.

- Chaque prise d'essai est faite en double et on ajoute dans tous les tubes $5 \mathrm{ml}$ du réactif acétique. Après mélange, les tubes sont bouchés par un tampon d'ouate ou mieux par un bouchon de verre si l'on dispose de tubes à cols rodés. Ils sont ensuite disposés dans un portoir, lequel est plongé dans un bain-marie bouillant. On maintient l'ébullition lente et régulière pendant 30 minutes. Le portoir contenant les tubes est alors déposé dans un bain d'eau courante froide, la réaction est ainsi stoppée par un refoidissement très rapide.

- Après un dernier mélange, les lectures s'effectuent au colorimètre en cuves carrées de $10 \mathrm{~mm}$ de côté. On choisit un filtre transmettant sous une longueur d'onde de $410 \mathrm{n}$. m. Le témoin blanc permet de régler le zéro de l'appareil.

\section{d. Expression des résultats}

Elle se fait en pourcentage de glucose dans le miel soit :

$$
100 \times \frac{\mathbf{P}^{1} \times \mathbf{E}^{2}}{\mathbf{P}^{2} \times \mathbf{E}^{1}}
$$


où

$\mathrm{P}^{\mathrm{I}}=$ poids du glucose dans la prise d'essai du témoin sucres réducteurs (en $\mu \mathrm{g}$ ).

$\mathbf{P}^{\mathbf{2}}=$ poids du miel dans la prise d'essai miel (en $\left.\mu \mathrm{g}\right)$.

$\mathrm{E}^{1}=$ lecture au colorimètre pour la prise d'essai du témoin sucres réducteurs $\left(e n D^{0}\right)$

$\mathrm{E}^{2}=$ lecture au colorimètre pour la prise d'essai miel $\left(\mathrm{en}^{\mathrm{0}}\right)$.

Lorsque $\mathrm{P}^{1}=150 \mu \mathrm{g}$ et $\mathrm{P}^{2}=400 \mu \mathrm{g}$ on a :

$$
100 \times \frac{150}{400}=37.5(1) \times \frac{\mathrm{E}^{2}}{\mathrm{E}^{1}}
$$

\section{e. Précautions à prendre}

Toute la verrerie utilisée et notamment les tubes à essais, doit être parfaitement propre, rincée à l'eau distillée et à l'alcool, séchée en étuve. Les tubes à essais sont en Pyrex ou autre qualité de verre résistant aux chocs thermiques provoqués par le passage du bain bouillant au bain froid.

Le pipetage du réactif acétique au moment du mélange est réalisé à l'aide d'une pipette automatique. Nous recommandons d'effectuer toutes les manipulations avec des gants pour se protéger de la solution corrosive et si possible de travailler sous hotte ventilée afin d'éviter une inhalation trop importante de la benzidine en milieu acétique. Enfin les parois extérieures des cuves sont soigneusement essuyées avant la lecture au colorimètre.

L'analyse se prête bien au travail en série; il convient cependant de réaliser au minimum deux témoins de sucres réducteurs dans chaque série.

\section{C. - Recherche du fructose}

\section{a. Principe}

On fait réagir le fructose contenu dans une solution de miel avec l'acide $\beta$ indolyl-acétique (A. B. I. A.) en milieu chlorhydrique concentré. Dans les conditions expérimentales décrites ci-dessous, on obtient une coloration violette proportionnelle à la concentration en fructose du milieu.

\section{b. Réactifs}

- I solution éthanolique à $0,5 \%$ d'acide $\beta$ indolyl-acétique.

- 2 acide chlorhydrique pur concentré.

\section{c. Mode opératoire}

On prélève à la micropipette $0,15 \mathrm{ml}$ de la solution de référence de sucres réducteurs, soit $30 \mu \mathrm{g}$ de fructose dans la prise d'essai. De la même manière on écoule $0,20 \mathrm{ml}$ de la solution de miel préparée pour le dosage précédent, soit $80 \mu \mathrm{g}$ de miel dans la prise d'essai. Un témoin à blanc est réalisé avec $0,20 \mathrm{ml}$ d'eau distillée.

Chaque prise d'essai est effectuée en double et on ajoute dans les tubes successivement $0,20 \mathrm{ml}$ de la solution d'acide $\beta$ indolyl-acétique, puis $8 \mathrm{ml}$ d'acide chlorhydrique. Après mélange les tubes sont bouchés, placés dans un portoir qui est plongé dans un bain thermostatique à $37^{\circ} \mathrm{C}$ pendant une heure. Ce temps écoulé le portoir contenant les tubes est déposé sans délai dans un bain d'eau courante froide, où il demeure pendant 5 minutes.

Après un dernier mélange les lectures s'effectuent au colorimètre en cuves carrées $(10 \times$ $10 \mathrm{~mm}$ ). On choisit un filtre transmettant sous une longueur d'ondes de $520 \mathrm{~nm}$.

\section{d. Expression des résultats}

Comme pour le dosage précédent, elle se fait en pourcentage de fructose contenu dans le miel soit :

$$
100 \times \frac{\mathrm{P}^{1} \times \mathrm{E}^{2}}{\mathrm{P}^{2} \times \mathrm{E}^{1}} \quad \text { ou } \quad \frac{100 \times 30}{80}=37.5(1) \times \frac{\mathrm{E}^{2}}{\mathrm{E}^{1}}
$$

\section{e. Remarques}

Elles sont identiques à celles qui concernent la recherche du glucose.

1. Ce qui constitue une approximation des teneurs moyennes en glucose et en fructose pour un miel; dans ces conditions les chiffres obtenus par $\mathrm{E}^{1}$ et $\mathrm{E}^{2}$ sont généralement voisins. 


\section{III. - RÉSULTATS}

Afin de tester la fiabilité de la méthode nous avons réalisé un certain nombre d'expériences de contrôle. En comparaison avec les deux sucres témoins (le glucose pour l'analyse à la benzidine et le fructose pour l'analyse à l'acide $\beta$ indolyl acétique), nous avons fait réagir successivement et à différentes concentrations quelques-uns des principaux sucres que l'on trouve dans les miels. Les résultats figurent aux tableaux 1 et 2 .

L'analyse à la benzidine révèle outre le glucose, un très faible pourcentage de fructose, $10 \%$ environ du maltose, ainsi que quelques polysaccharides qui réagissent partiellement mais dont la proportion totale dans le miel excède rarement 2 à $3 \%$. Comme élément de comparaison, nous avons introduit dans cette étude le dosage chimique du glucose par l'iode. Au cours de cette analyse le glucose réagit normalement; mais réagissent aussi $6 \%$ environ du fructose témoin ainsi qu'un quart du maltose qui est en règle générale le disaccharide le plus abondant dans le miel (de 4 à $7 \%$ en moyenne).

L'analyse colorimétrique à l'acide $\beta$ indolyl acétique permet de révéler le fructose; cependant quelques traces de glucose, moins de $5 \%$ du maltose et $50 \%$ du saccharose réagissent. Mais exception faite pour 2 ou 3 types de miels, le produit de l'abeille contient très peu de saccharose ( 1 à $1,5 \%$ en moyenne).

Au tableau 3, nous présentons des résultats traduits en pourcentage de glucose, de fructose et de sucres réducteurs; ces résultats sont obtenus par différentes méthodes d'analyses. Si nous considérons comme analyse de référence le dosage par chromatographie en phase gazeuse, nous pouvons faire les quelques observations suivantes.

En ce qui concerne le glucose, les résultats obtenus par colorimétrie sont assez voisins de la référence, sauf pour le miellat. Par contre le dosage chimique révèle toujours un pourcentage de glucose exagéré. Les résultats de l'analyse colorimétrique du fructose se situent à des taux supérieurs à ceux du témoin de référence. Enfin, les résultats, exprimés en sucres réducteurs, obtenus en additionnant le glucose et le fructose révélés par les analyses colorimétriques sont très voisins des résultats d'un dosage réductimétrique classique (type Bertrand). Les écarts avec les résultats obtenus par chromatographie, sont, ici encore relativement importants. 
TENEUR EN GLUCOSE ET EN FRUCTOSE DES MIELS

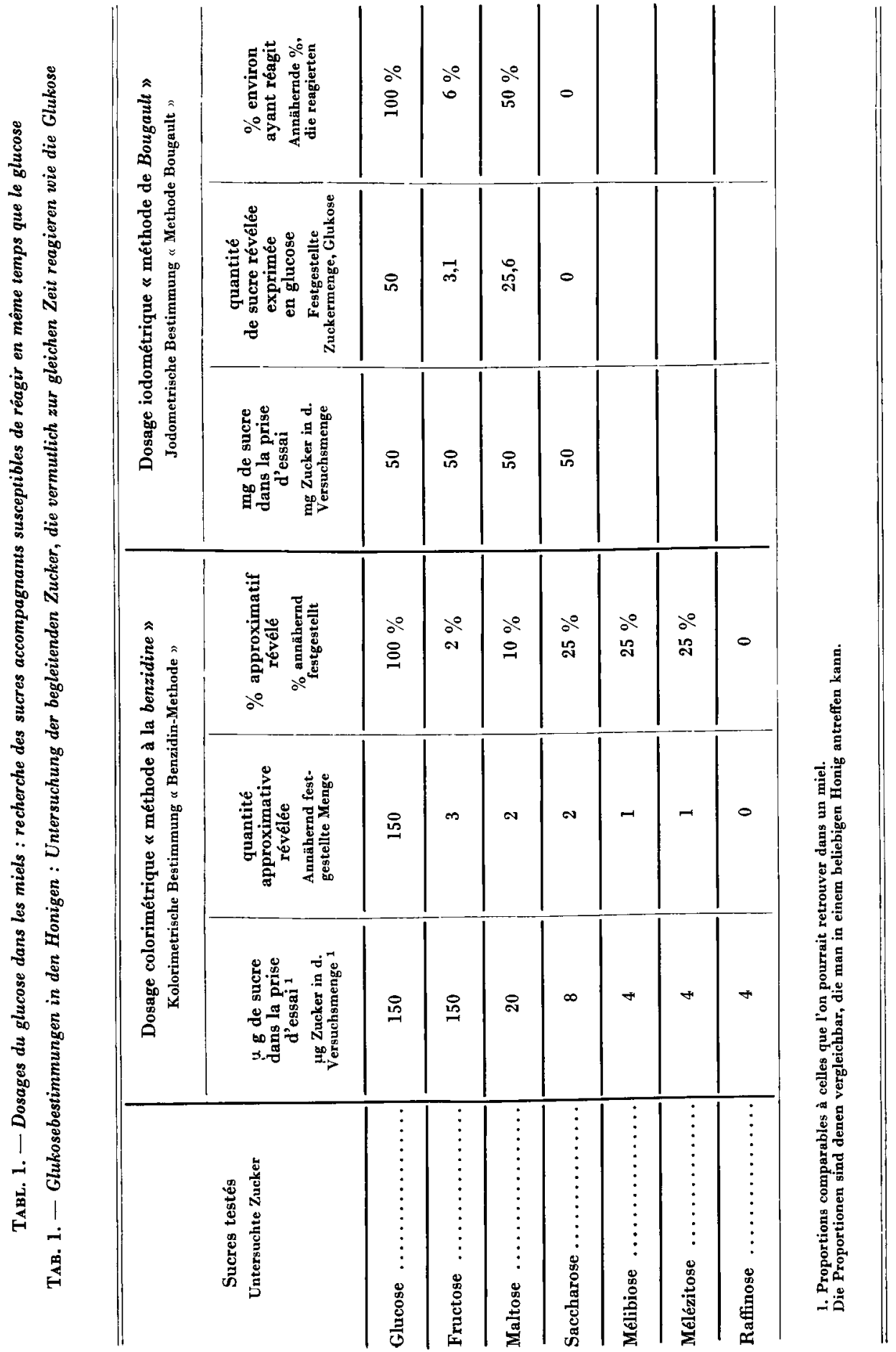


TABL. 2. - Dosage du fructose dans les miels :

recherche des sucres accompagnants succeptibles de réagir en même temps que le fructose

TAB. 2. - Fruktose-Bestimmung in den Honigen :

Untersuchung der begleitenden Zucker, die vermutlich gleichzeitig mit der Fruktose reagieren

\begin{tabular}{|c|c|c|c|}
\hline \multirow[b]{2}{*}{$\begin{array}{c}\text { Sucres testés } \\
\text { Untersuchte Zucker }\end{array}$} & \multicolumn{3}{|c|}{$\begin{array}{l}\text { Dosage colorimétrique Méthode à l'A. B. I. A. } \\
\text { Kolorimetrische Bestimmung - A.B.I.A. Methode }\end{array}$} \\
\hline & $\begin{array}{l}\text { '2g. de sucre dans } \\
\text { la prise d'essai }{ }^{1} \\
\text { Mg Zucker i. d. } \\
\text { Versuchsmenge }\end{array}$ & $\begin{array}{c}\text { quantité approxi- } \\
\text { mative révélée } \\
\text { Annähernd } \\
\text { festgestellte Menge }\end{array}$ & $\begin{array}{l}\% \text { approximatif } \\
\text { révélé } \\
\text { Annähernde \%, d. } \\
\text { festgestellt wurden }\end{array}$ \\
\hline Fructose.................. & 30 & 30 & $100 \%$ \\
\hline Glucose $\ldots \ldots \ldots \ldots \ldots \ldots$ & 30 & 0,2 & $0,6 \%$ \\
\hline Maltose $\ldots \ldots \ldots \ldots \ldots \ldots \ldots$ & 4 & 0,2 & $5 \%$ \\
\hline Saccharose $\ldots \ldots \ldots \ldots \ldots \ldots$ & 1,6 & 0,9 & $60 \%$ \\
\hline Mélébiose $\ldots \ldots \ldots \ldots \ldots \ldots$ & 0,8 & $\mathbf{0}$ & 0 \\
\hline Mélézitose $\ldots \ldots \ldots \ldots \ldots \ldots$ & 0,8 & 0,2 & 25 \\
\hline Raffinose $\ldots \ldots \ldots \ldots \ldots \ldots \ldots$ & 0,8 & 0,2 & $25 \%$ \\
\hline
\end{tabular}

1. Dans des proportions comparables à celles que l'on pourrait retrouver dans un miel. In Proportionen, die denen vergleichbar sind, die man in einem beliebigen Honig antreffen könnte.

\section{IV. - DISCUSSION}

En conclusion, les méthodes d'analyses que nous décrivons autorisent une estimation beaucoup plus qu'un dosage précis des deux principaux sucres réducteurs du miel. Toutefois, cette estimation est bien meilleure que celle obtenue par d'autres méthodes de chimie courante, principalement pour la recherche du glucose. Avec ce dernier sucre, d'ailleurs, et par la méthode colorimétrique que nous décrivons ici, le pourcentage de l'erreur commise dans les dosages que nous avons effectués varie entre 0 et $2 \%$ en plus, ceci par rapport à l'analyse de référence. Cet excès est dû à la réaction partielle de quelques polysaccharides contenus dans le miel. La réaction d'un faible pourcentage de fructose comme le prouve l'analyse au tableau 1 ne fausse pas les résultats car elle est compensée par l'utilisation d'un témoin contenant glucose et fructose pour parts égales. 
वे

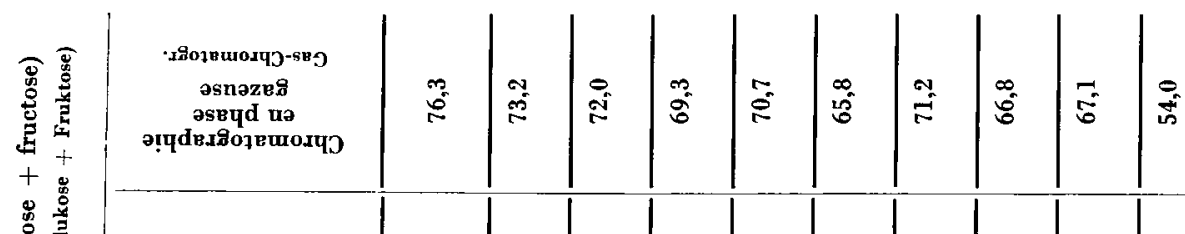

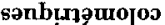
seseso

窇

苍

总

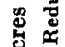

कี วำ

purdparag

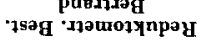

« puespag "

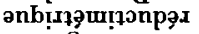
ว8eso

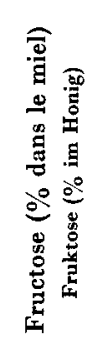

-18078uoryว-849

วsnวze⿳⺈

วseyd $u$

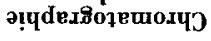

\begin{tabular}{l|l|l|l|l}
0 & 0 & 0 & $m$ & 0 \\
$n$ & 0 & 5 & 0
\end{tabular}

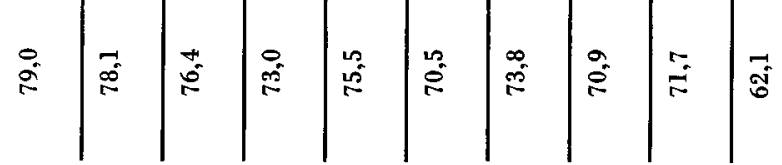

V I I $\mathbf{H} \cdot \mathbf{H}$

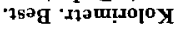

" $\left.\mathbf{V}^{*} \mathrm{I} \cdot \mathrm{G} \cdot \mathrm{V}\right)$

วnb!xวน!xо [оว

วक्लs०प

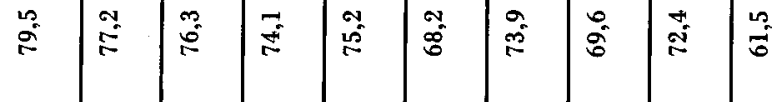

\begin{tabular}{|c|c|c|c|c|c|c|c|c|c|c|c|}
\hline \multirow{3}{*}{ 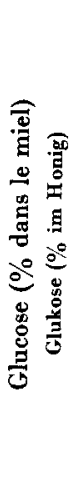 } & 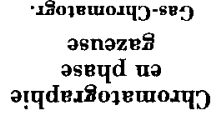 & م્ & 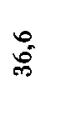 & $\stackrel{0}{\stackrel{0}{n}}$ & 总 & ๙ิ & $\stackrel{b}{\stackrel{b}{\sim}}$ & 㤐 & $\stackrel{\overrightarrow{0}}{\dot{m}}$ & ڤ్ & 今ึ \\
\hline & 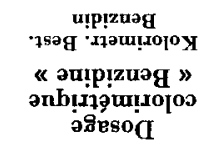 & $\stackrel{10}{\circ}$ & $\begin{array}{l}n \\
5 \\
5\end{array}$ & 煦 & $\begin{array}{l}\text { fi } \\
\text { fin }\end{array}$ & 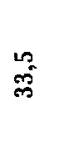 & 周 & 㪇 & $\frac{10}{n}$ & $\frac{3}{5}$ & స్ \\
\hline & 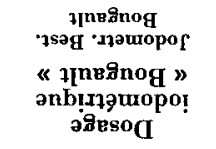 & $\stackrel{\vec{*}}{+}$ & 岁 & g. & 苞 & 悉 & $\tilde{m}^{\infty}$ & $\underset{-\infty}{+\infty}$ & की & $\overrightarrow{\hat{\rho}}$ & के \\
\hline & 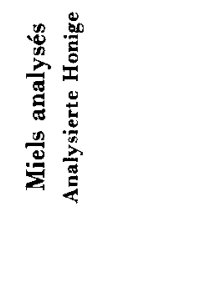 & 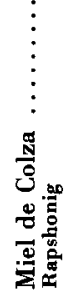 & 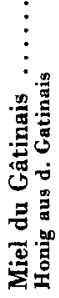 & 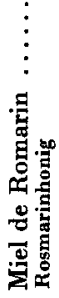 & 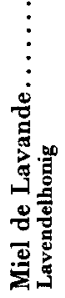 & 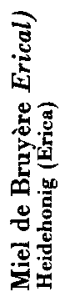 & 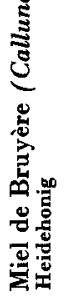 & 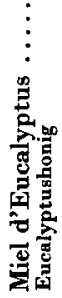 & 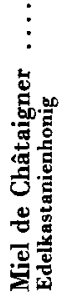 & 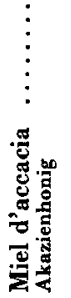 & 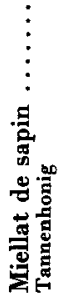 \\
\hline
\end{tabular}


L'estimation que nous pouvons faire de la teneur en fructose du miel est moins précise que la précédente et cependant les principaux sucres autres que le fructose ne réagissent pas, ou très faiblement, lors du dosage. Pour cette analyse la marge d'erreur que nous observons se situe entre 2 à $5 \%$ en excès par rapport à la référence. C'est une constatation que nous n'expliquons pas et sur laquelle nous ne pouvons formuler qu'une hypothèse. Il est possible que le fructose témoin, produit de l'intervertion chimique du saccharose, réagisse avec l'acide $B$ indolyl acétique de manière sensiblement différente du fructose $d u$ miel qui est, pour partie, le produit de l'intervertion biochimique due à la gluco-invertase sécrétée par l'abeille. Nous avons fait un témoin de référence avec du saccharose interverti par voie biochimique; nous disposions pour cela d'une préparation d'invertase du commerce. Dans ce cas les résultats ont été identiques à ceux obtenus par l'intervertion chimique. Nous avons finalement retenu cette dernière technique car elle est plus simple à utiliser.

Enfin, si nous comparons les résultats des deux estimations colorimétriques groupées, ils correspondent aux résultats obtenus par un dosage réductimétrique couramment utilisé pour les analyses de miel. Dans ce domaine, donc, la précision relative des analyses colorimétriques est équivalente à celle d'un dosage chimique classique.

Sur le plan de l'utilisation pratique des résultats l'approximation obtenue par ces méthodes colorimétriques apparaît généralement suffisante. Ajoutons encore comme élément positif que ces analyses peuvent être réalisées en séries et que les colorations obtenues sont relativement stables au moins pendant le temps des lectures. Il est préférable cependant de ne pas utiliser ces techniques pour l'analyse des sucres réducteurs dans les miellats de sapin. Ces derniers peuvent contenir de 15 à $20 \%$ de polysaccharides et, comme nous l'avons montré, certains de ces sucres réagissent en partie et faussent les résultats. Par contre, nous conseillons ce type d'analyse comme technique d'utilisation courante, particulièrement en ce qui concerne la recherche du glucose dans les miels en vue de la préparation des opérations de conditionnement du miel.

Dans tous les cas où l'on aura besoin de résultats très précis la chromatographie en phase gazeuse restera, bien entendu, la méthode de référence.

Reçu pour publication en novembre 1972.

Eingegangen im November 1972.

\section{REMERCIEMENTS}

Nous tenons à remercier M. Pountallier, Ingénieur chimiste au Laboratoire de Recherches Apicoles de Nice, qui a réalisé pour nous les dosages par chromatographie en phase gazeuse. 


\section{ZUSAMMENFASSUNG}

Das Kristallisieren des Honigs sowie seine gewerbliche Behandlung, die daranf abzielt, seine einwandfreie Erhaltung zu gewährleisten, stellen Probleme, deren Lösung zum grossen Teil von der Beschaffenheit des Produktes und ganz besonders von seinem Gehalt an Glukose und Fruktose abhängt. Ziel dieser Arbeit ist es, praktische und schnelle Analysen-Methoden aufzuzeigen, die es erlauben, den prozentualen Anteil jedes dieser beiden reduzierenden Zucker unter den Arbeitsbedingungen im Labor einer Imkergenossenschaft oder eines bedeutenden Honighändlers zu bestimmen.

Die Bestimmung des Glukosegehaltes erfolgt durch eine kolorimetrische Methode, die Benzidin in saurem Milieu benutzt, während der Fruktose-Anteil durch eine andere kolorimetrische Methode festgestellt wird : hierbei wird die Analyse in einem Milieu von konzentrierter Salzsäure in Anwesenheit von B-Indolyl-Essigsäure durchgeführt. Zur Kontrolle wird eine entsprechende Bestimmung von Glukose und Fruktose mittels Gas-Chromatographie durchgeführt (Tab. 3).

Die Ergebnisse sind in bezug auf die kolorimetrische Bestimmung der Glukose zufriedenstellend; im Vergleich zur Kontrollmethode zeigen sie nur einen leichten Überschuss, der auf die Teilreaktionen einiger im Honig vorhandener Polysaccharide zurückzuführen ist (Tab. 1). Bei der Analyse der Fruktose sind die Ergebnisse weniger genau. Das Abweichen von der Kontrolle besteht in einem wesentlich höheren Überschuss als im vorangegangenen Versuch, obwohl die hauptsächlichen begleitenden Zucker wenig reagieren (Tab. 2). Auf jeden Fall aber ist die Genauigkeit der aufgezeichneten Resultate identisch mit oder besser als die der klassischen chemischen Analysen zur Qualitätskontrolle des Honigs (Tab. 3).

Die in dieser Arbeit beschriebenen Analysen-Methoden gewährleisten eine sichere Bestimmung des Gehalts an den reduzierenden Zuckern Fruktose und vor allem Glukose im Honig. Doch die Gas-Chromatographie bleibt die beste Methode, wenn eine grosse Genauigkeit erforderlich ist, was in der Regel bei Forschungsarbeiten oder beim Erstellen von Gutachten zutrifft.

Dagegen dürften in der Praxis, wenn es darum geht, die handelsüblichen Eigenschaften eines Honigs zu kontrollieren, die Werte genau genug sein, um dem für die Behandlung des Honigs Verantwortlichen die notwendigen Aufschlüsse zu geben.

\section{RÉFÉRENCES BIBLIOGRAPHIQUES}

Herovsky A., 1956. Dosage colorimétrique des cétoses par l'acide $B$ indolyl acétique (en tchèque), chem. Listy., 50, 1593-1598.

Jones J.-K., Pridham J.-B., 1954. A colorimètric estimation of sugars using Benzidine. Biochem. J., 55, 288-290.

Maurizio A., 1950. Papierchromatographische Untersuchungen an Blütenhonigen und Nektar. Ann. Abeille, 4, 291-341.

Pourtallier J., 1954. Détermination par chromatographie en couche mince des sucres du miel. Bull. Apicole, VII (2), 197-211.

Pourtallier J,, 1967. Détermination quantitative des sucres des miels par chromatographie en phase gazeuse. Bull. Apicole X (2), 209-212.

White J.-W., MaHER J., 1954. Sélective absorption méthod for détermination of the sugars of honey. Journ. A. O. A. C., 37 (2), 466-478.

White J.-W., 1962. Composition of american Honeys. U.S. départ. Agric. Techn. Bull., 1261, I, 124 . 